\title{
Quantitative genetic basis of floral design in a natural plant
}

\section{population}

Juannan Zhou ${ }^{1,4}$, Charles B. Fenster ${ }^{2}$, Richard J. Reynolds ${ }^{3}$

${ }^{1}$ Simons Center for Quantitative Biology, Cold Spring Harbor Laboratory, Cold Spring Harbor, NY 11724 USA

${ }^{2}$ Department of Biology and Microbiology, South Dakota State University, Brookings, SD 57007

USA

${ }^{3}$ Division of Clinical Immunology and Rheumatology, Department of Medicine, University of Alabama at Birmingham, AL 35294 USA

${ }^{4}$ Author for Correspondence

${ }^{4}$ Email: jzhou@cshl.edu 


\begin{abstract}
The amount of genetic variation of floral traits and the degree to which they are genetically correlated are important parameters for the study of plant evolution. Estimates of these parameters can reveal the effect of historical selection relative to neutral processes such as mutation and drift, and allow us to predict the short-term evolutionary trajectory of a population under various selective regimes. Here, we assess the heritability and genetic correlation of the floral design of a native N. American tetraploid plant, Silene stellata (Caryophyllaceae), in a natural population. Specifically, we use a linear mixed model to estimate the genetic parameters based on a genealogy reconstructed from highly variable molecular markers.

Overall, we found significant heritabilities in five out of nine studied traits. The level of heritability was intermediate $(0.027-0.441)$. Interestingly, the floral trait showing the highest level of genetic variation was previously shown to be under strong sexually conflicting selection, which may serve as a mechanism for maintaining the observed genetic variation. Additionally, we also found prevalent positive genetic correlations between floral traits. Our results suggest that S. stellata is capable of responding to phenotypic selection on its floral design, while the abundant positive genetic correlations could also constrain the evolutionary trajectories to certain directions. Furthermore, this study demonstrates the utility and feasibility of marker-based approaches for estimating genetic parameters in natural populations.
\end{abstract}




\section{Introduction}

A major goal in evolutionary biology is to predict the phenotypic changes of populations under the force of selection. This requires, along with the estimate of selection pressures, detailed understanding of the underlying genetic variation of quantitative traits as well as genetic correlations between traits, as illustrated by the multivariate breeder's equation (Lande and Arnold, 1983). Furthermore, the pattern of existing genetic variation also reveals the efficacy of historical selection relative to genetic drift, mutation and migration (Lynch and Walsh, 1998).

Since the application of the breeder's equation to evolutionary studies (Lande and Arnold 1983), extensive efforts have been dedicated to studying the levels of genetic variation in morphological and life history characters in plants (e.g., Ashman and Majetic 2006). Because floral diversity is believed to reflect, in part, pollinator mediated selection(Fenster et al., 2004), and mating system evolution is linked to floral evolution (Barrett, 1998), documentation of within population genetic variation of floral traits has been a historic focus. A survey by Ashman and Majetic (2006) based on studies conducted mostly in controlled environments found an average $h^{2}$ of 0.39 . Although it seems that there is considerable standing genetic variation in floral traits to allow evolutionary response to pollinator-mediated selection (Ashman and Majetic, 2006) and other forces such as herbivores (Strauss and Whittall, 2006), genetic parameters estimated in controlled environments should be interpreted with caution, since plants in general show large phenotypic plasticity (Primack and Kang, 1989), and estimation of heritability in controlled environments could be potentially biased upward due to the lower environmental effects, as well as G×E interactions (Coyne and Beecham, 1987; Conner et al., 2003).

Traditional methods of heritability estimation rely on the covariation of breeding values between close relatives and usually require construction of experimental populations with uniform family structures, e.g. parent-offspring, full-sib, and half-sib designs (Falconer and Mackay, 1996). A large sample size is also required to ensure statistical power (Mitchell-Olds and Rutledge, 1986). Therefore, the cost and labor of this type of breeding program pose significant obstacles to the study of genetic variation in wild plant populations. 
Recently, a restricted maximum likelihood (REML) approach termed the "animal model” has been applied to investigate genetic bases of complex traits in wild populations (Kruuk, 2004; Wilson et al., 2010). The animal model is more flexible in that it does not require a fixed family structure in the study population and is able to take into account all relationships in any pedigree. The animal model is therefore expected to provide more statistical power than close-kin comparisons (Kruuk, 2004). It is also less susceptible to confounding effects such as inbreeding, selection and shared environment (Kruuk and Hadfield, 2007), and is more robust to unbalanced datasets than parent-offspring regression approaches (Lynch et al., 1998; Kesson et al., 2008). Given all these advantages, one limitation of the animal model in its application to field studies is the requirement of a fully known pedigree, which is generally very difficult to acquire in natural populations, especially for organisms with complex life history and mating systems.

Therefore, despite its success in animal and plant breeding, application of the animal model to evolutionary studies has been relatively rare and mostly has been restricted to bird and large mammal populations that are either under long-term surveillance, or shows behaviors that allow the inference of relatedness between individuals (Milner et al. 2000, Kruuk 2004, Kesson et al. 2008, Akesson et al. 2008). In contrast, the availability of highly variable molecular marker and various statistical methods now makes it possible to implement the animal model with genealogies reconstructed based on individual genotypes (Frentiu et al., 2008; Blonk et al., 2010; Frère et al., 2010). This approach has the potential to expand the range of application of the animal model to wild populations and has been shown to perform well in several animal systems (Frentiu et al., 2008; Blonk et al., 2010; Frère et al., 2010).

In this paper, we focus on a hermaphroditic plant, Silene stellata, native to eastern North America. S. stellata is an emerging model organism for studying pollinator-plant interaction and the evolution of mutualism (Kephart et al., 2006; Bernasconi et al., 2009) due to its complex interaction with the pollinator/seed predator moth Hadena ectypa. A previous study has shown prevalent phenotypic selection on the S. stellata floral traits (Zhou et al., 2020). Most notably, the petal dimensions of S. stellata were found to be under strong conflicting selection through the two sexual functions of the hermaphroditic plant, mediated by the positive and negative interaction with H. ectypa. 
Here, we determine the genetic variation as well as the genetic correlation of the S. stellata floral design in a natural population of S. stellata, based on a genealogy reconstructed using 8 highly variable microsatellite markers. We aim to examine the genetic basis of the $S$. stellata floral design in light of the previously observed phenotypic selection, and to answer three main questions about the evolutionary biology of S. stellata: (1) Is the genetic variation consistent with the pattern of selection observed previously? In particular, do the traits under conflicting selection show elevated levels of genetic variation? (2) Is the study S. stellata population able to evolve under directional selection? (3) Do the genetic correlation between traits restrict the evolutionary trajectory dictated by the previously observed multivariate phenotypic selection?

\section{METHODS}

\section{Study population}

We focus on a natural population of Silene stellata near the University of Virginia's Mountain Lake Biological Station in Giles County, Virginia, U.S.A. (37.3471, -80.5426 , elevation $\approx 1,100-1,300$ meters). Silene stellata L. is an infrequent, iteroparous, long-lived perennial herb that is distributed throughout the eastern half of the United States. In our Virginia study sites. S. stellata flowering occurs from early July through early September and is characterized by panicle inflorescences with white, hermaphroditic, protandrous flowers (average of $25 \pm 2.5$ ovules/pistil, Reynolds et al. 2009). An individual plant at our study site usually produces multiple stems and on average produces $\sim 40$ flowers each flowering season (Reynolds et al., 2012). Outcrossing rate is relatively high based on estimates from both pollinator exclusion experiments (>73\%, Reynolds 2008) and mating system estimation using microsatellite markers ( 83\%, Zhou et al. 2018b).

S. stellata exhibits a complex pollination biology, whereby a nocturnal moth, Hadena ectypa pollinates the $S$. stellata flowers during its adult stage, but also predates large quantities of S. stellata reproductive tissue during its larval stage. The Silene-Hadena interaction is further complicated by a guild of generalist 
pollinator moths, that are equally effective at pollen transfer (Reynolds et al., 2012). In a previous study, we found that the parasitic and mutualistic interaction between $H$. ectypa leads to conflicting selection on the petal dimension through the two sexual functions of $S$. stellata (Zhou et al., 2020). Furthermore, the transition between $H$. ectypa and the copollinating species across the flowering phenology also leads to temporally variable selection.

We measured S. stellata plants in four cohorts in 2012 and 2013. In each year, two cohorts of flowering plants were measured in the course of a week during both the early and late flowering season with a oneweek separation between experimental periods. We sampled different plants for each cohort and in each year to avoid pseudo-replication, yielding a total number of 227 plants $(2012$ early, $\mathrm{N}=59 ; 2012$ late, $\mathrm{N}=$ 58; 2013 early, $\mathrm{N}=55 ; 2014$ late, $\mathrm{N}=55$ ). All study plants were within a 20-by-20-meter exclosure constructed in a natural S. stellata population near Mountain Lake Biological Station $\left(37.348296^{\circ},-\right.$ $80.544301^{\circ}$, elevation $\left.\approx 1,100-1,300 \mathrm{~m}\right)$. The two cohorts within a year correspond to periods when $H$. ectypa or the copollinators were the dominant pollinator, respectively (Zhou, Dudash, and Fenster, 2016; Zhou et al., 2020). Our sampling of genotypes to quantify patterns of genetic variation thus take into account potentially different selection pressures that plants in the early and late cohorts experience.

In contrast to the predominant diploidy of Silene species distributed in the Old World, the vast majority of N. American Silene have been shown to be polyploids, including tetra-, hexa-, and octoploids, with tetraploids being the most common type (Popp and Oxelman, 2007). The N. American polyploid Silene species have at least two independent origins. Phylogenetic relationships within the clade containing $S$. stellata are poorly resolved, possibly due to rapid evolution, recombination among homoeologues, and homoplasy (Popp and Oxelman, 2007). The status of (auto- vs. allo-) tetraploidy of S. stellata is not well understood. However, the maximum number of alleles amplified per locus was consistently four across all 8 markers in this study and we found no evidence of fixed heterozygotes, supporting a hypothesis of autotetraploidy (Parisod et al., 2010). 


\section{Phenotypic traits}

We quantified the pattern of genetic variation and covariation for nine floral characters: (1) corolla tube length, TL; (2) corolla tube width, TW; (3) largest petal length, PL; (4) largest petal width, PW; (5) number of fringes on the distal margin of the petal, FR; (6) anther exsertion, AN; (7) stigma exsertion, ST, (see

Figure 1). We measured the distance from the nectary at the base of the flower to the tip of the anther or the stigma and calculated AN and ST as the difference between nectary-anther or nectary-stigma distance and corolla tube length. All floral traits except ST were measured on flowers during the first day of opening (the male phase). Stigma measurements were taken in the first day of the female phase. We counted fringe number (FR) on a randomly chosen petal in which we measured the other six floral traits. Additionally, we recorded the total number of flowers (NF) and floral display height (HT). Measurements were carried out with calipers $(0.1 \mathrm{~mm})$ during the course of one week on each plant for PL, PW, FR, TL, TW, ST, AN. Display height was measured as the height of the highest flower to the nearest $\mathrm{cm}$ using a meter stick, while NF was quantified by counting the number of flowers open throughout the experimental period. On average five flowers per plant were measured for floral traits (total number of flowers measured, mean number measured per plant: 2012 early, $\mathrm{N}=280,4.75 ; 2012$ late, $\mathrm{N}=239,4.12 ; 2013$ early, $\mathrm{N}=294,5.35 ; 2014$ late, $\mathrm{N}=281,5.11)$.

\section{Genotyping and genealogy reconstruction}

DNA was extracted using the Autogen Plant Kit, following the manufacturer's protocol. Plants were genotyped on 8 novel microsatellite loci designed for S. stellata (Zhou, Dudash, Fenster, et al., 2016). Individual genotypes were visualized and manually scored using GeneMapper V3.7 software (Applied Biosystems). There are two main approaches to replace the known pedigree in the animal model. First, one can construct a matrix, $\mathbf{A}$, consisting of relatedness coefficients that are independently estimated for all pairs of individuals. Since the relatedness coefficients are estimated independently for each pair of individuals, compatibility between dyads is not guaranteed (e.g. in examining the relationships between three individuals, A, B and C, the pairs A-B and A-C might be inferred as full-sibs, while the pair B-C might be inferred as half- or non-sib). This incompatibility can lead to the non-positive-definiteness of $\mathbf{A}$, 
resulting in its failure to be inverted for the subsequent REML analysis (Frentiu et al., 2008). Therefore, we implemented the simulated annealing approach using the program COLONY (Jones and Wang, 2010) to reconstruct a genealogy containing all study plants that ensured statistical consistency. COLONY uses the full-pedigree likelihood approach by maximizing the likelihood of the entire pedigree structure rather than individual relationships. Therefore it uses genotype data in a more efficient way and also avoids the troublesome compatibility issue (Jones and Wang, 2010). Additionally, it allows the simultaneous inference of parentage and sibship, which is desirable for our purpose given the extended age structure in the $S$. stellata population (Dudash and Fenster unpublished data). Using COLONY, we generated a genealogy in which all pairs of individuals were classified as full sibs, half sibs, parent-offspring, or unrelated. Since less related pairs of individuals will not be reflected in the estimated genealogy, some information in the data is inherently lost. However, the advantage of genealogy reconstruction is that all pairwise relationships are inherently consistent, and the pedigree data format is compatible with all REML software.

Given that $S$. stellata is a tetraploid, the allelic dosage of partial heterozygotes cannot be readily resolved (for example, an individual showing two alleles $\mathrm{A}, \mathrm{B}$ on a given locus can have AAAB, AABB or $\mathrm{ABBB}$ as the underlying genotype). Additionally, most programs of genealogy reconstruction do not support polyploid data. In the face of these technical difficulties, we transformed the tetraploid genotypes following the protocol described in Wang and Scribner (2014) for application in polyploids. Specifically, we converted the tetraploid genotypes at a codominant microsatellite locus with $k$ alleles to diploid phenotypes at $k$ dominant "loci", following the method of Rodzen et al. ( 2004). For example, for a locus with four alleles (A1, A2, A3, A4), an individual with alleles A1, A2, will be coded as $\{1,1,0,0\}$ at four pseudo-diploid loci, and an individual with alleles A1, A2, A3 will be coded as $\{1,1,1,0\}$, and so forth (See Supplemental Table 1 for a detailed example). This approach has been demonstrated to accurately infer sibship, parentage and selfing rate from a typical set of microsatellite markers (Wang and Scribner, 2014).

To assess the reliability of the genealogy reconstructed using the above procedure, we also generated a matrix consisting of independent estimates of pairwise relatedness coefficients, $r$, using the package POLYRELATEDNESS 1.6 (Huang et al., 2014), designed for autopolyploids. POLYRELATEDNESS uses 
a maximum likelihood procedure to estimate the vector $\Delta=\left[\Delta_{1} \Delta_{2} \Delta_{3} \Delta_{4}\right.$, for every pairwise comparison, where $\Delta_{\mathrm{i}}$ is the probability that two individuals share $i$ alleles that are Identical-By-Descent (IBD). The relatedness coefficient $r$ is then calculated as $r=\sum_{i=0}^{4} i \Delta_{i} / 4$. We converted the genealogy generated by COLONY to a relatedness matrix using the statistical package "kinship2" (Therneau and Sinnwell, 2015) in R (R Core Team, 2016). We then compared the two matrices generated by POLYRELATEDNESS and COLONY by running a mantel test in R with 9999 permutations and found a significant correlation (Pearson correlation coefficient $r=0.665, p<0.001$ ).

\section{Mixed model analysis}

Univariate variance component analysis was carried out in the REML program Wombat (Meyer, 2007). We fit the following repeated-measure animal model for the seven floral traits using data pooled across years with year and season as fixed effects: $y=X b+Z_{a} a+Z_{c} c+e$, where $y$ is the vector of observed phenotypic values, $b$ is the vector containing the main effects of year (2012 vs. 2013) and season (early vs. late), $a$ is the vector containing individual additive genetic effects, c represents the vector of permanent environmental effects, and $e$ contains the residuals. $X, Z_{a}$, and $Z_{c}$ are design matrices relating appropriate fixed and random effects to individual records. The multiple measurements of a floral trait on a given plant were grouped under the same permanent environmental effect potentially including micro-environmental, maternal, dominance, and epistatic effects on each individual. Permanent environmental effects were not included in the model for number of flowers (NF) and display height (HT), for which only one record was available per plant. The total phenotypic variance $V_{P}$ is therefore partitioned into additive genetic variance $\left(V_{A}\right)$, variance due to permanent environmental effect $\left(V_{P E}\right)$, and residual variance $\left(V_{E}\right)$. Narrow-sense heritabilities were then calculated using the estimated additive genetic variance as $h^{2}=V_{A} /\left(V_{A}+V_{P E}+\right.$ $\left.V_{E}\right)$. We also reported the repeatability estimates for the seven floral traits as $h^{2}=\left(V_{A}+V_{P E}\right) /\left(V_{A}+V_{P E}+\right.$ $\left.V_{E}\right)$. For comparative purposes, we also report the coefficient of variation $(\mathrm{CV})$, i.e., $100 \times$ additive genetic variance divided by the phenotypic mean.

We specified Wombat to sample 100000 data points from the multivariate normal distribution of ML estimates generated at convergence, to approximate the standard errors for the parameter estimates. 


\section{Genetic correlations}

Additive correlations, $r_{A}$ were estimated using the multivariate version of the animal model described above. The model included only traits for which we observed significant additive genetic components in previous univariate analyses, since $r_{A}$ is ill-defined if one trait has heritability equal to zero because the genetic correlation between trait $x$ and $y$ is defined as: $r_{A}(x, y)=\frac{\operatorname{Cov}_{A}(x, y)}{\sqrt{\operatorname{Var}_{A}(x) \operatorname{Var}_{A}(y)}}$, where $\operatorname{Cov}_{A}(x, y)$ is the genetic covariance (Lynch and Walsh, 1998; Coltman et al., 2005), and . Additionally, we also report phenotypic correlations between pairs of traits based on the outputs of Wombat.

\section{Correlation of environmental effects between relatives}

Since previous work has shown that pollen dispersal distance between plants is as low as $1-2 \mathrm{~m}$ (Reynolds et al., 2009; Zhou, Zimmer, et al., 2018) and seeds of S. stellata mainly disperse passively through gravity, closely related plants could experience common environmental effects due to spatial autocorrelation, which could potentially inflate our genetic component estimates. To rule out this possibility, we compared the relatedness matrix A generated using COLONY with the matrix D which contained pairwise Euclidean distances calculated based on individual GPS coordinates, by running a mantel test with 9999 permutations in R (R Core Team, 2016). No significant correlation was found between the two matrices (Pearson correlation coefficient $\mathrm{r}=-0.048, p>0.999$ ).

\section{Statistical tests}

We assessed the significance levels of individual parameter estimates as well as comparison of estimates across years by calculating the $z$ score, equal to the difference between two estimates divided by the square root of the sum of the squared standard errors. For the purpose of testing significance of single estimates against zero, the formula is reduced to the ratio between the estimate and its standard error. $P$ values of the $z$ scores were calculated from a large sample standard normal distribution. We also quantified the association in estimates of $h^{2}, r_{\mathrm{A}}$ between years using Pearson's product-moment correlation $(r)$. 
Additionally, a Mantel test with 9999 permutations was used to compare the two $r_{\mathrm{A}}$ matrices for 2012 and 2013. All comparisons were performed in R ( $\mathrm{R}$ Core Team, 2016).

\section{Results}

\section{Heritability and variance components}

We first fit separate REML models for 2012 and 2013. For both years, PW is the only trait with significant heritability $\left(2012: h^{2}=0.530, p=0.06 ; 2013: h^{2}=0.530, p=0.004\right)$. Heritability estimates were similar for all traits across years (Supplemental Table 2; Supplemental Figure 1). Therefore, in the subsequent univariate analyses we pooled the data across years and employed a REML model with year as a fixed effect.

Using the pooled dataset, we found five floral traits (PL, FR, TL, TW, and PW) with significant $h^{2}(p<$ $0.05)$, while $h^{2}$ of anther exsertion (AN) was marginally significant $(p=0.085) . . h^{2}$ values ranged from 0 $(\mathrm{NF}, p>0.99)$ to 0.441 (PL, $p<0.001)$. Genetic coefficients of variation $(\mathrm{CV})$ ranged between $0.266(\mathrm{NF})$ and 14.049. (PL). Repeatabilities were significant in all cases $(p<0.05)$ and ranged from 0.391 (ST) to 0.885 (PL) (Table 1).

Genetic correlations

Similar to univariate analyses, we first fit the multivariate animal model for each year independently, then compared the estimates between years. Since no significant differences occurred between years (Supplemental Table 3; Supplemental Figure 2), we pooled the data across years and performed the multivariate animal model using the full model with year as a fixed effect.

Genetic correlations calculated using the pooled dataset were positive in 14 out of 15 comparisons and significant in nine of them (mean $r_{\mathrm{A}}=0.236 \pm 0.295 \mathrm{SE}$, Table 2). All significant $r_{\mathrm{A}}$ 's were positive and ranged between 0.324 and 0.972 . The highest $r_{\mathrm{A}}$ was TL vs. AN $\left(r_{\mathrm{A}}=0.972 \pm 0.175 \mathrm{SE}, p<0.001\right)$, 
followed by PL vs. PW $\left(r_{\mathrm{A}}=0.944 \pm 0.260 \mathrm{SE}, p<0.001\right)$. Petal fringe (FR) was only significantly correlated with PW $\left(h^{2}=0.324 \pm 0.161\right.$ SE, $\left.p<0.05\right)$.

Similar to genetic correlations, values of phenotypic correlations $r_{\mathrm{p}}$ were positive except for TL vs. TW $\left(r_{\mathrm{P}}=-0.208 \pm 0.053 \mathrm{SE}, p<0.001\right)$ (Table 2). Mean $r_{\mathrm{p}}=0.2538 \pm 0.202 \mathrm{SD}$. The only insignificant $r_{\mathrm{p}}$ was TL vs. FR $\left(r_{\mathrm{P}}=0.08 \pm 0.055 \mathrm{SE}, p>0.05\right)$. The average squared genetic correlation $\left(r^{2} \mathrm{~A}\right)$ was 0.28 , while the average squared phenotypic correlation $\left(r^{2}{ }_{\mathrm{P}}\right)$ was 0.10 (Cheverud 1988). A Mantel test with 999 permutations revealed significant correlation between the genetic and phenotypic correlation matrices (Pearson correlation coefficient $r=0.91, p<0.001$ ).

\section{Discussion}

Although statistically more powerful and flexible than traditional methods such as parent-offspring regression, the requirement of a known pedigree has impeded the application of the animal model to natural plant populations. There have been a number of studies that attempted to replace the known pedigree with one reconstructed from molecular genotypes (Frentiu et al. 2008, Blonk et al. 2010, Frère et al. 2010).

These results generally support the efficacy of the pedigree-free approach in estimating genetic components. Here we reconstructed a genealogy for 227 S. stellata individuals based on 11 highly variable microsatellite loci and observed prevalent genetic variation as well as covariation in the floral traits of $S$. stellata.

One major challenge in using molecular genealogy in an animal model analysis is the statistical error in the inference of pairwise relationship due to the limitation of genetic markers and the genealogy reconstruction algorithm, because the pedigree is assumed by the model to be known without error. Little empirical work has been conducted to examine how erroneous relationship inference affects the animal model. However, we used a microsatellite marker set that was highly polymorphic (mean number of alleles per locus $=22.6$ ). Additionally, we observed a fair range of relatedness including unrelated, half-sib, full- 
sib and parent-offspring in the reconstructed genealogy. The high resolution of our marker set and the variation in relatedness may help to minimize the effect of erroneous relationships on our inference of genetic parameters (Ritland, 1996; Frentiu et al., 2008).

We observed significant heritabilities, $h^{2}$ in five out of nine traits of S. stellata. Additionally, anther exsertion (AN) was marginally significant $\left(h^{2}=0.257 \pm 0.149 \mathrm{SD}, p=0.085\right)$. The $h^{2}$ 's were intermediate (0.027-0.441) for all the remaining floral traits. Among all floral traits, stigma exsertion (ST) had the lowest heritability $\left(h^{2}=0.027 \pm 0.131 \mathrm{SD}, p>0.10\right)$. One likely explanation for this low $h^{2}$ other than strong historical selection, is that the protandrous $S$. stellata flowers transition to the female phase after the one-day male-phase through slow extension of the style. Thus, although we made all measurements of ST on flowers during the first day of the female stage, the exact time of measurements and possible asynchrony in growth rates of the styles could introduce additional environmental variation. We did not find significant $h^{2}$ in display height (HT) and number of flowers (NF). One likely cause for the lack of genetic components in NF and HT could be that S. stellata is a long-lived perennial, and phenotypic variation in these two traits mainly reflects variation in plant age and local microhabitat, rather than the underlying genetic factors.

Our mean estimate of $h^{2}$ over all nine traits was 0.27 , while mean $h^{2}$ of the seven floral traits was 0.32 . These estimates were lower than the mean of 0.39 reported by Ashman and Majetic (2006). This is not surprising, as $66 \%$ percent of the studies surveyed by Ashman and Majetic (2006) were conducted in controlled environments and high environmental variance in field conditions might contribute to our lower observed $h^{2}$. Additionally, in a previous study, we also observed substantial neutral genetic variation in three local S. stellata populations (Zhou, Dudash, et al., 2018). Our assessment of genetic variation in morphological traits here seems to be concordant with this previous finding, although there is generally a weak relationship between levels of variation of neutral markers and quantitative traits (Reed et al., 2002).

In a previous study (Zhou et al., 2020), we found significant directional selection on several floral traits of S. stellata, including petal length, petal width, and fringe number, as well as vegetative traits including number of flower and display height. Our finding of prevalent heritability in the S. stellata floral traits 
indicate the potential for short-term evolutionary response to contemporary selective pressures. On the other hand, although number of flowers were consistently under the strongest positive selection, the absence of genetic variation of this trait precludes its response to the observed selective pressure.

Importantly, in Zhou et al. (2020), we also found conflicting selection pressures exerted on the petal dimensions through the two sexual functions. Remarkably, the floral trait under the strongest conflicting selection (petal length) is also found to have the highest heritability in this paper. Additionally, an earlier multi-year study of the same $S$. stellata population showed that certain floral traits were under phenotypic selection significant only in certain years (Zhou et al., 2020). Together, this indicates that the prevalent additive genetic variation in our study population could have resulted from conflicting selection exerted through different fitness components of the plant and/or the temporal fluctuation of selective pressures. Finally, while G×E interactions could also be responsible for the maintenance of genetic variation (Gillespie and Turelli, 1989), the high similarities in $h^{2}$ and $r_{\mathrm{A}}$ across years do not seem to support this explanation.

We found significant genetic correlations, $r_{\mathrm{A}}$ in nine out of 15 pairwise comparisons. We found a mean $r_{\mathrm{A}}$ of 0.45 , which is higher than Ashman and Majetic (2006) (Mean $\left.\pm \mathrm{ST}=0.33 \pm 0.03\right)$. Following Cheverud (1988), we compared the phenotypic and genetic correlation matrices using the average squared correlation coefficients. The average squared genetic correlation was 0.28 , while the average squared phenotypic correlation was 0.10 . This difference is very close to the mean difference of 0.20 reported by Cheverud (1988), and may be caused by sampling error given the limitation of a pedigree-free animal model. Alternatively, the observed differences could be caused by significant environmental variation in the natural environment (Willis et al., 1991), since the majority of cases studied by Cheverud (1988) were conducted in laboratory or agricultural environments.

The significant $r_{\mathrm{A}}$ 's were uniformly positive. This suggests that the floral design of $S$. stellata could potentially respond harmoniously to natural selection for flower size. On the other hand, divergence of character states through selection may be constrained along axes determined by the abundant genetic correlations (Falconer, 1981). Specifically, genetic correlations between traits associated with pollinator 
attraction (PL, PW, FR in this study) (Bell, 1985; Ashman and Majetic, 2006; Mitchell et al., 2015) and traits associated with pollen transfer (TL, TW, AN, ST in this study) (Nilsson, 1988; Campbell et al., 1991, 1996; Ashman and Majetic, 2006; Reynolds et al., 2010) were significant in six of nine cases. These positive genetic correlations could hamper independent evolution of different functional modules under potentially opposing selection pressures by various selective agents (e.g., mutualists vs. antagonists) and through the male vs. female function of the hermaphroditic flower (Strauss and Whittall, 2006; Steven et $a l ., 2007)$. In contrast, the lack of genetic correlation between certain pairs of floral traits could provide additional minor axes for divergence of character states. For example, the $r_{\mathrm{A}}$ between corolla tube length (TL) and width (TW) is close to zero $\left(r_{\mathrm{A}}=-0.062 \pm 0.138 \mathrm{ST}, p>0.10\right)$ while both traits had significant $h^{2}$. This indicates that these two dimensions of the S. stellata corolla tube could potentially respond independently to opposing selection pressures.

In conclusion, our results show the study S. stellata population exhibit prevalent moderate genetic variation in its floral design, which can be attributed to the previously observed conflicting and variable selection regime. This shows the study population is capable of responding to directional selection in the face of environmental change, whereas the genetic correlation between traits could also impose evolutionary constraints in certain multivariate selection scenarios. 
Table 1. Heritabilities $\left(h^{2}\right)$ and repeatabilities $\left(r^{2}\right)$ of nine morphological traits of $S$. stellata, with corresponding standard errors $(\mathrm{SE}), z$ scores $(z)$, and significance from zero $(p)$. Parameters were estimated with data pooled across two years including year as a fixed effect, using the univariate animal model. $\mathrm{CV}_{\mathrm{A}}$ : coefficient of variation of additive genetic variance.

\begin{tabular}{|c|c|c|c|c|c|c|c|c|c|}
\hline Trait & $\overline{h^{2}}$ & $\mathrm{SE} \mathrm{h}^{2}$ & $\mathrm{z} \mathbf{h}^{2}$ & $\overline{p \mathbf{h}^{2}}$ & $\overline{\mathbf{r}^{2}}$ & $\mathrm{SE} \mathbf{r}^{2}$ & $\overline{z \mathbf{r}^{2}}$ & $\overline{p \mathbf{r}^{2}}$ & $\overline{C V_{A}}$ \\
\hline PL & 0.441 & 0.041 & 10.756 & 0.000 & 0.885 & 0.058 & 15.393 & 0.000 & 2.570 \\
\hline PW & 0.357 & 0.047 & 7.596 & 0.000 & 0.675 & 0.049 & 13.787 & 0.000 & 2.260 \\
\hline FR & 0.360 & 0.047 & 7.660 & 0.000 & 0.720 & 0.051 & 14.073 & 0.000 & 2.312 \\
\hline TL & 0.367 & 0.042 & 8.738 & 0.000 & 0.870 & 0.054 & 16.243 & 0.000 & 2.462 \\
\hline TW & 0.424 & 0.043 & 9.860 & 0.000 & 0.882 & 0.060 & 14.762 & 0.000 & 2.519 \\
\hline ST & 0.027 & 0.131 & 0.206 & 0.837 & 0.391 & 0.136 & 2.867 & 0.004 & 0.453 \\
\hline $\mathbf{A N}$ & 0.257 & 0.149 & 1.725 & 0.085 & 0.577 & 0.147 & 3.917 & 0.000 & 1.449 \\
\hline HT & 0.172 & 0.212 & 0.811 & 0.417 & $-{ }^{a}$ & - & - & - & 0.896 \\
\hline NF & 0.000 & 0.176 & 0.000 & 1.000 & - & - & - & - & 0.010 \\
\hline
\end{tabular}

${ }^{a} r^{2}$ only available for traits with multiple measurements per plant.PL: petal length; PW: petal width; FR:

number of petal fringes; TL: corolla tube length; TW: corolla tube width; ST: stigma exsertion; AN: anther exsertion; HT: display height; NF: number of flowers. 
Table 2. Phenotypic correlations ( $r_{P}$, above the diagonal), additive genetic correlations ( $r_{A}$, below the diagonal), with corresponding standard errors (in parentheses) among six floral traits of S. stellata, calculated with data pooled across years using the multivariate animal model with year as a fixed effect. Significance levels were calculated for individual $z$ scores based on a large sample standard normal distribution ( $* * * P<0.001 ; * * P<0.01 ; * P<0.05$; ns, not significant). PL: petal length; PW: petal width; FR: number of petal fringes; TL: corolla tube length; TW: corolla tube width; ST: stigma exsertion; AN: anther exsertion; HT: display height; NF: number of flowers.

\begin{tabular}{|c|c|c|c|c|c|c|c|}
\hline & & \multicolumn{3}{|l|}{ Attraction } & \multicolumn{3}{|l|}{ Pollen transfer } \\
\hline & & PL & PW & FR & $\mathrm{TL}$ & TW & AN \\
\hline \multirow[t]{3}{*}{ Attraction } & PL & - & $\begin{array}{l}0.559(0.047) \\
* * *\end{array}$ & $\begin{array}{l}0.19(0.056) \\
* * *\end{array}$ & $\begin{array}{l}0.388(0.052) \\
* * *\end{array}$ & $\begin{array}{l}0.39(0.049) \\
* * *\end{array}$ & $\begin{array}{l}0.277(0.049) \\
* * *\end{array}$ \\
\hline & PW & $\begin{array}{l}0.944(0.260) \\
* * *\end{array}$ & - & $\begin{array}{l}0.142 \\
(0.054) * *\end{array}$ & $\begin{array}{l}0.208(0.05) \\
* * *\end{array}$ & $\begin{array}{l}0.527(0.043) \\
* * *\end{array}$ & $\begin{array}{l}0.353(0.045) \\
* * *\end{array}$ \\
\hline & FR & $\begin{array}{l}0.310(0.187) \\
\text { ns }\end{array}$ & $\begin{array}{l}0.324(0.161) \\
*\end{array}$ & - & $\begin{array}{l}0.08(0.055) \\
\text { ns }\end{array}$ & $\begin{array}{l}0.116(0.054) \\
*\end{array}$ & $\begin{array}{l}0.141(0.051) \\
* *\end{array}$ \\
\hline \multirow[t]{3}{*}{$\begin{array}{l}\text { Pollen } \\
\text { transfer }\end{array}$} & TL & $\begin{array}{l}0.466(0.198) \\
*\end{array}$ & $\begin{array}{l}0.337(0.145) \\
*\end{array}$ & $\begin{array}{l}0.178 \\
(0.145) \mathrm{ns}\end{array}$ & - & $\begin{array}{l}-0.208 \\
(0.053) * * *\end{array}$ & $\begin{array}{l}0.487(0.039) \\
* * *\end{array}$ \\
\hline & TW & $\begin{array}{l}0.774(0.257) \\
* *\end{array}$ & $\begin{array}{l}0.663(0.177) \\
* * *\end{array}$ & $\begin{array}{l}0.283 \\
(0.165) \mathrm{ns}\end{array}$ & $\begin{array}{l}-0.062 \\
(0.138) \mathrm{ns}\end{array}$ & - & $\begin{array}{l}0.157(0.05) \\
* *\end{array}$ \\
\hline & AN & $\begin{array}{l}0.626(0.204) \\
* *\end{array}$ & $\begin{array}{l}0.474(3.12) \\
* *\end{array}$ & $\begin{array}{l}0.296 \\
(0.160) \mathrm{ns}\end{array}$ & $\begin{array}{l}0.972(0.175) \\
* * *\end{array}$ & $\begin{array}{l}0.163(0.143) \\
\text { ns }\end{array}$ & - \\
\hline
\end{tabular}


bioRxiv preprint doi: https://doi.org/10.1101/2020.11.06.371831; this version posted January 14, 2021. The copyright holder for this preprint (which was not certified by peer review) is the author/funder. All rights reserved. No reuse allowed without permission.

Figure 1. Diagram of Silene stellata floral traits measured. All floral traits except stigma exertion were measured on flowers during the first day of opening (the male phase, panel A and B). Stigma exsertion was measured on the first day of the female phase (C).

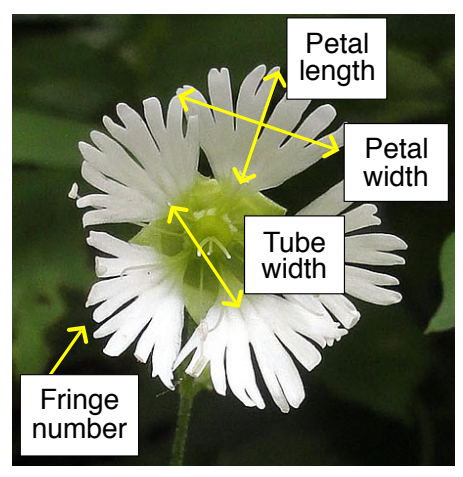

A

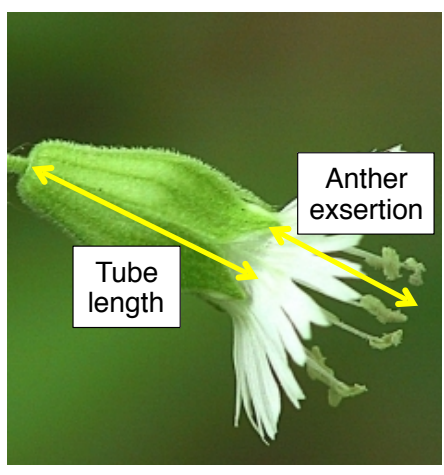

B

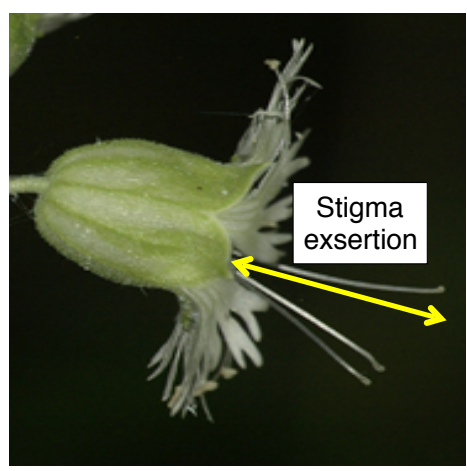

C 
Supplemental Table 1. Sample pseudo-diploid input file containing actual genotypes of 10 individuals at locus 3R with 20 alleles.

\begin{tabular}{|l|l|l|l|l|l|l|l|l|l|l|l|l|l|l|l|l|l|l|l|l|l|l|l|l|}
\hline ID & \multicolumn{2}{l|}{$\begin{array}{l}\text { Original } \\
\text { tetraploid } \\
\text { genotype }\end{array}$} \\
\hline
\end{tabular}


Supplemental Table 2. Heritabilities $\left(h^{2}\right)$ and corresponding standard errors (SE), $z$ scores $(z)$, and significance from zero ( $p$ ) of nine morphological traits of S. stellata estimated for year 2012 and 2013 based on 11 microsatellite loci, using the univariate animal model. Also shown are the $z$ scores and significance levels $(p)$ for comparison of $h^{2}$ across years. Significance levels were calculated for individual $z$ scores based on a large sample standard normal distribution. PL: petal length; PW: petal width; FR:

number of petal fringes; TL: corolla tube length; TW: corolla tube width; ST: stigma exsertion; AN: anther exsertion; HT: display height; NF: number of flowers.

\begin{tabular}{|c|c|c|c|c|c|c|c|c|c|c|}
\hline Trait & 2012 & & & & 2013 & & & & $2012 \mathrm{v}$ & 2013 \\
\hline & $h^{2}$ & SE & $z$ & $p$ & $h^{2}$ & SE & $z$ & $p$ & $z$ & $p$ \\
\hline PL & 0.366 & 0.599 & 0.611 & 0.541 & 0.339 & 0.319 & 1.063 & 0.288 & 0.040 & 0.968 \\
\hline PW & 0.530 & 0.284 & 1.866 & 0.062 & 0.538 & 0.185 & 2.908 & 0.004 & -0.024 & 0.981 \\
\hline FR & 0.344 & 0.495 & 0.695 & 0.487 & 0.363 & 0.350 & 1.037 & 0.300 & -0.031 & 0.975 \\
\hline TL & 0.351 & 0.427 & 0.822 & 0.411 & 0.310 & 0.289 & 1.073 & 0.283 & 0.080 & 0.937 \\
\hline TW & 0.332 & 0.335 & 0.991 & 0.322 & 0.350 & 0.277 & 1.264 & 0.206 & -0.041 & 0.967 \\
\hline ST & 0.000 & 0.251 & 0.000 & 1.000 & 0.001 & 0.334 & 0.003 & 0.998 & -0.002 & 0.998 \\
\hline AN & 0.437 & 0.385 & 1.135 & 0.256 & 0.411 & 0.270 & 1.522 & 0.128 & 0.055 & 0.956 \\
\hline HT & 0.052 & 0.389 & 0.134 & 0.894 & 0.044 & 0.399 & 0.110 & 0.912 & 0.014 & 0.989 \\
\hline $\mathbf{N F}$ & 0.000 & 0.332 & 0.000 & 1.000 & 0.089 & 0.382 & 0.233 & 0.816 & -0.176 & 0.860 \\
\hline
\end{tabular}


Supplemental Table 3. Genetic correlations $\left(r_{A}\right)$ and corresponding standard errors (SE), $z$ scores and significance from zero $(p)$ between six morphological traits of S. stellata estimated for year 2012 and 2013 , using the multivariate animal model. Also shown are the $z$ scores and significance levels for comparisons of $r_{A}$ across years. Significance levels were calculated for individual $z$ scores based on a large sample standard normal distribution. PL: petal length; PW: petal width; FR: number of petal fringes; TL: corolla tube length; TW: corolla tube width; AN: anther exsertion.

\begin{tabular}{|c|c|c|c|c|c|c|c|c|c|c|}
\hline & \multicolumn{4}{|l|}{2012} & \multicolumn{4}{|l|}{2013} & \multicolumn{2}{|c|}{2013 vs. 2013} \\
\hline & $r_{A}$ & SE & $z$ & $p$ & $r_{A}$ & SE & $z$ & $p$ & $z$ & $p$ \\
\hline PL vs. PW & 0.933 & 0.762 & 1.224 & 0.221 & 0.915 & 0.417 & 2.194 & 0.028 & 0.021 & 0.983 \\
\hline PL vs. FR & 0.406 & 0.483 & 0.841 & 0.401 & 0.354 & 0.314 & 1.127 & 0.260 & 0.090 & 0.928 \\
\hline PL vs. TL & 0.340 & 0.448 & 0.759 & 0.448 & 0.350 & 0.354 & 0.989 & 0.323 & -0.018 & 0.986 \\
\hline PL vs. TW & 0.852 & 0.788 & 1.081 & 0.280 & 0.860 & 0.509 & 1.690 & 0.091 & -0.009 & 0.993 \\
\hline PL vs. AN & 0.525 & 0.451 & 1.164 & 0.244 & 0.551 & 0.357 & 1.543 & 0.123 & -0.045 & 0.964 \\
\hline PW vs. FR & 0.380 & 0.354 & 1.073 & 0.283 & 0.312 & 0.237 & 1.316 & 0.188 & 0.160 & 0.873 \\
\hline PW vs. TL & 0.365 & 0.343 & 1.064 & 0.287 & 0.308 & 0.233 & 1.322 & 0.186 & 0.137 & 0.891 \\
\hline PW vs. TW & 0.657 & 0.387 & 1.698 & 0.090 & 0.665 & 0.280 & 2.375 & 0.018 & -0.017 & 0.987 \\
\hline PW vs. AN & 0.417 & 0.254 & 1.642 & 0.101 & 0.410 & 0.210 & 1.952 & 0.051 & 0.021 & 0.983 \\
\hline FR vs. TL & 0.158 & 0.293 & 0.539 & 0.590 & 0.186 & 0.257 & 0.724 & 0.469 & -0.072 & 0.943 \\
\hline FR vs. TW & 0.386 & 0.421 & 0.917 & 0.359 & 0.338 & 0.286 & 1.182 & 0.237 & 0.094 & 0.925 \\
\hline FR vs. AN & 0.319 & 0.337 & 0.947 & 0.344 & 0.267 & 0.225 & 1.187 & 0.235 & 0.128 & 0.898 \\
\hline TL vs. TW & -0.091 & 0.266 & -0.342 & 0.732 & -0.069 & 0.229 & -0.301 & 0.763 & -0.063 & 0.950 \\
\hline TL vs. AN & 0.916 & 0.606 & 1.512 & 0.131 & 0.949 & 0.486 & 1.953 & 0.051 & -0.042 & 0.966 \\
\hline TW vs. AN & 0.238 & 0.267 & 0.891 & 0.373 & 0.222 & 0.215 & 1.033 & 0.302 & 0.047 & 0.963 \\
\hline
\end{tabular}


Supplemental Figure 1. Association between heritabilities $\left(h^{2}\right)$ estimated for year 2012 and 2013, using the univariate animal model. Pearson correlation coefficient $\mathrm{r}=0.879, p<0.01$; Spearman rank correlation $\varrho=$ 0.854, $p<0.01$. PL: petal length; PW: petal width; FR: number of petal fringes; TL: corolla tube length; TW: corolla tube width; ST: stigma exsertion; AN: anther exsertion; HT: display height; NF: number of flowers.

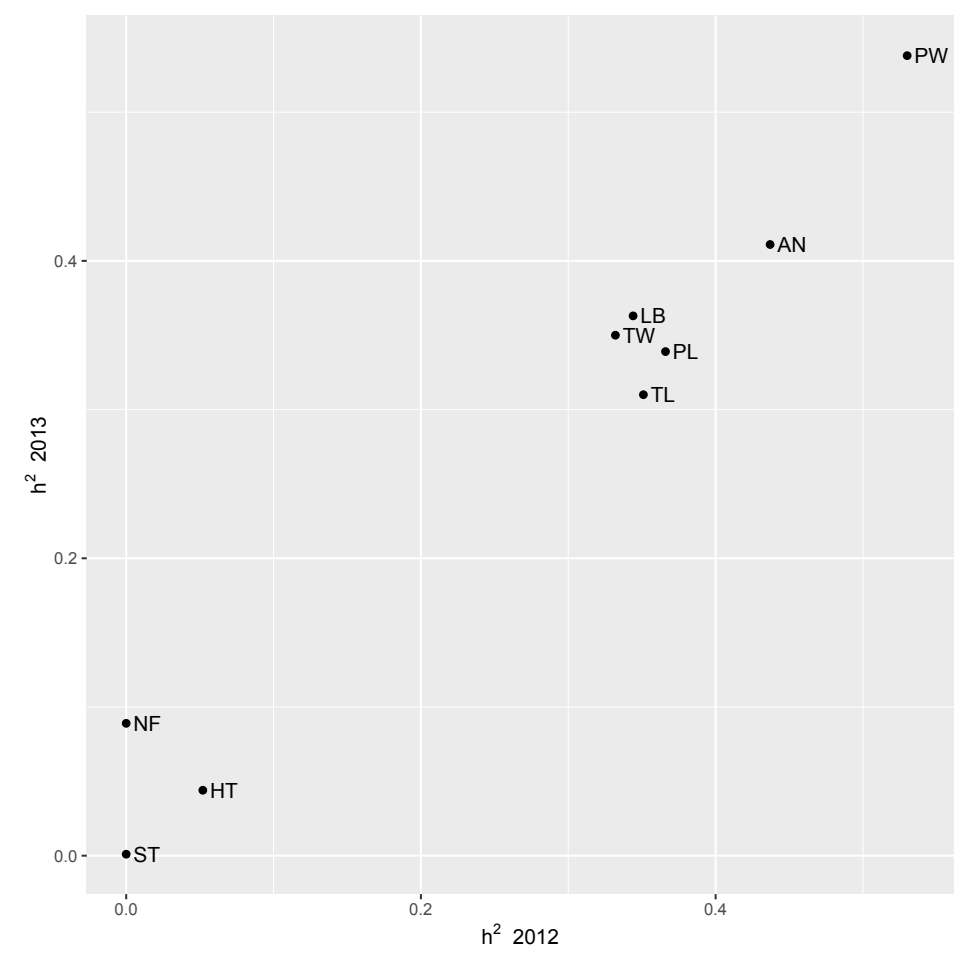


Supplemental Figure 2. Association between genetic correlations $\left(r_{A}\right)$ estimated for year 2012 and 2013 using the multivariate animal model. Pearson correlation coefficient $\mathrm{r}=0.978, p<0.001$; Spearman rank correlation $\varrho=0.975, p<0.001$. PL: petal length; PW: petal width; FR: number of petal fringes; TL: corolla tube length; TW: corolla tube width; ST: stigma exsertion; AN: anther exsertion; HT: display height; NF: number of flowers.

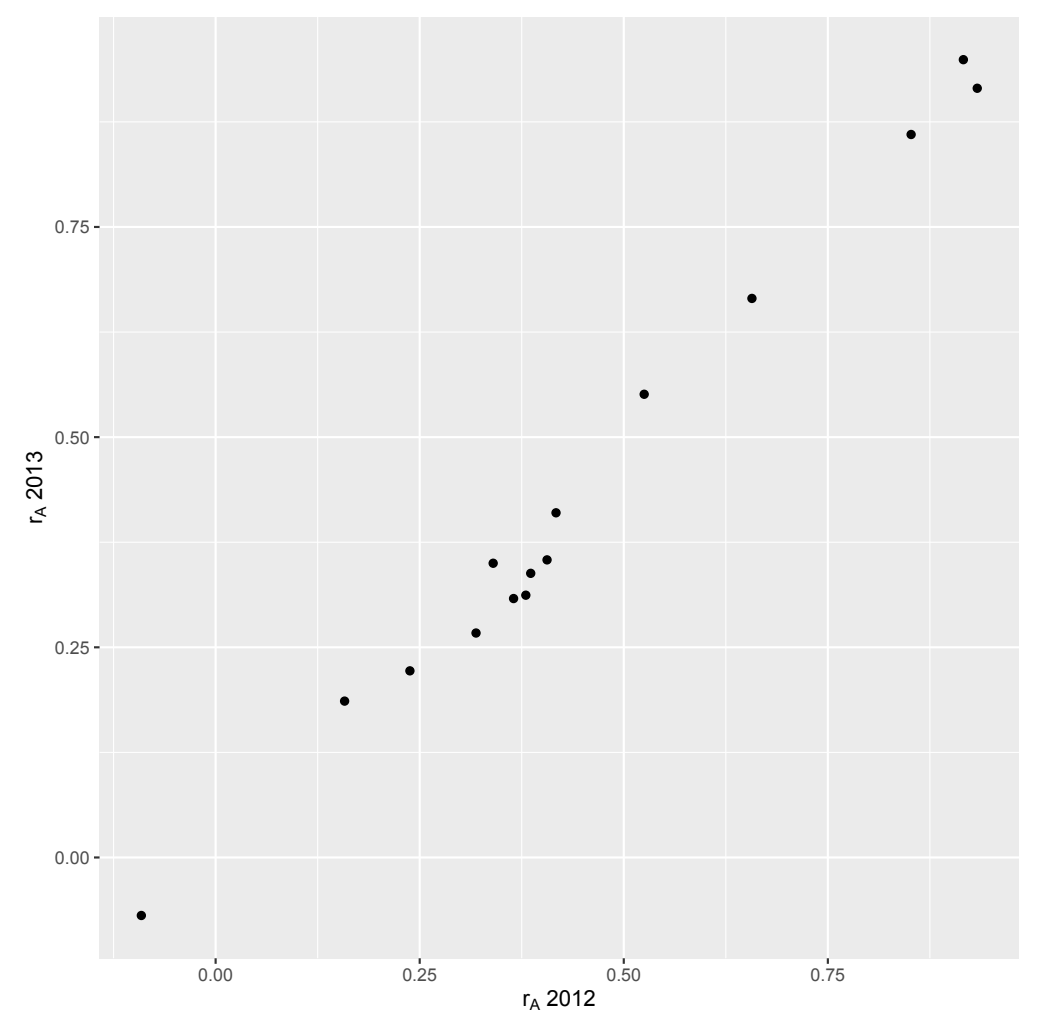




\section{Bibliography}

Akesson M, Bensch S, Hasselquist D, Tarka M, Hansson B (2008). Estimating heritabilities and genetic correlations: comparing the 'animal model' with parent-offspring regression using data from a natural population. PLoS One 3: e1739.

Ashman T-L, Majetic CJ (2006). Genetic constraints on floral evolution: a review and evaluation of patterns. Heredity (Edinb) 96: 343-52.

Barrett SCH (1998). The evolution of mating strategies in flowering plants. Trends Plant Sci 3: 335-341.

Bell G (1985). On the function of flowers. Proc R Soc London B Biol Sci 224: 223-265.

Bernasconi G, Antonovics J, Biere A, Charlesworth D, Delph LF, Filatov D, et al. (2009). Silene as a model system in ecology and evolution. Heredity (Edinb) 103: 5-14.

Blonk RJW, Komen H, Kamstra A, van Arendonk J a M (2010). Estimating breeding values with molecular relatedness and reconstructed pedigrees in natural mating populations of common sole, Solea solea. Genetics 184: 213-9.

Campbell DR, Waser NM, Price M V. (1996). Mechanisms of Hummingbird-Mediated Selection for Flower width in Ipomopsis Aggregata. Ecology 77: 1463-1472.

Campbell DR, Waser NM, Price M V, Lynch EA, Mitchell RJ (1991). Components of phenotypic selection: pollen export and flower corolla width in Ipomopsis aggregata. Evolution (N Y) 45: 1458-1467.

Cheverud JM (1988). A comparison of genetic and phenotypic correlations. Evolution (N Y) 42: 958-968.

Coltman DW, O’Donoghue P, Hogg JT, Festa-Bianchet M (2005). Selection and genetic (co)variance in bighorn sheep. Evolution (N Y) 59: 1372-1382.

Conner JK, Franks R, Stewart C (2003). Expression of additive genetic variances and covariances for wild radish floral traits: comparison between field and greenhouse environments. Evolution (N Y) 57: 487-495.

Coyne JA, Beecham E (1987). Heritability of two morphological characters within and among natural populations of Drosophila melanogaster. Genetics 117: 727-737.

Falconer DS (1981). Introduction to quantitative genetics. Longman.

Falconer DS, Mackay TFC (1996). Introduction to quantitative genetics, 4th edn. Longman: Harlow.

Fenster CB, Armbruster WS, Wilson P, Dudash MR, Thomson JD (2004). Pollination syndromes and floral specialization. Annu Rev Ecol Evol Syst 35: 375-403.

Frentiu FD, Clegg SM, Chittock J, Burke T, Blows MW, Owens IPF (2008). Pedigree-free animal models: the relatedness matrix reloaded. Proc R Soc London, Ser B Biol Sci 275: 639-47.

Frère CH, Krützen M, Mann J, Connor RC, Bejder L, Sherwin WB (2010). Social and genetic interactions drive fitness variation in a free-living dolphin population. Proc Natl Acad Sci U S A 107: 19949-54.

Gillespie JH, Turelli M (1989). Genotype-environment interactions and the maintenance of polygenic variation.

Genetics 121: 129-138.

Huang K, Guo ST, Shattuck MR, Chen ST, Qi XG, Zhang P, et al. (2014). A maximum-likelihood estimation of pairwise relatedness for autopolyploids. Heredity (Edinb): 1-10.

Jones OR, Wang J (2010). COLONY: A program for parentage and sibship inference from multilocus genotype data. Mol Ecol Resour 10: 551-555.

Kephart S, Reynolds RJ, Rutter MT, Fenster CB, Dudash MR (2006). Pollination and seed predation by moths on Silene and allied Caryophyllaceae: evaluating a model system to study the evolution of mutualisms. New Phytol 169: 667-80.

Kesson MÅ, Bensch S, Hasselquist D, Tarka M, Hansson B, Gardner A (2008). Estimating Heritabilities and Genetic Correlations: Comparing the 'Animal Model' with Parent-Offspring Regression Using Data from a Natural Population.

Kruuk LEB (2004). Estimating genetic parameters in natural populations using the 'animal model'. Proc R Soc London, Ser B Biol Sci 359: 873-90.

Kruuk LEB, Hadfield JD (2007). How to separate genetic and environmental causes of similarity between relatives. $J$ Evol Biol 20: 1890-1903.

Lande R, Arnold SJ (1983). The Measurement of Selection on Correlated Characters. Evolution (N Y) 37: 1210.

Lynch M, Walsh B (1998). Genetics and analysis of quantitative traits.

Lynch M, Walsh B, others (1998). Genetics and analysis of quantitative traits. Sinauer Sunderland, MA.

Meyer K (2007). WOMBAT - A tool for mixed model analyses in quantitative genetics by restricted maximum 
likelihood (REML). J Zhejiang Univ Sci B 8: 815-821.

Milner JM, Pemberton JM, Brotherstoneà S, Albon SD (2000). Estimating variance components and heritabilities in the wild : a case study using the 'animal model' approach. 13: 804-813.

Mitchell-Olds T, Rutledge JJ (1986). Quantitative genetics in natural plant populations: a review of the theory. Am Nat 127: 379-402.

Mitchell TC, Dötterl S, Schaefer H, Heilmeier H (2015). Hawkmoth pollination and elaborate petals in Cucurbitaceae : The case of the Caribbean endemic Linnaeosicyos amara. Flora 216: 50-56.

Nilsson LA (1988). The evolution of flowers with deep corolla tubes. Nature 334: 147-149.

Parisod C, Holderegger R, Brochmann C (2010). Evolutionary consequences of autopolyploidy. New Phytol 186: 5-17. Popp M, Oxelman B (2007). Origin and Evolution of North American Polyploid Silene (Caryophyllaceae). Am J Bot 94: 33-349.

Primack RB, Kang H (1989). Measuring fitness and natural selection in wild plant populations. Annu Rev Ecol Syst 20: 367-396.

R Core Team (2016). R: A Language and environment for statistical computing.

Reed DH, Briscoe DA, Frankham R (2002). Inbreeding and extinction: the effect of environmental stress and lineage. Conserv Genet 3: 301-307.

Reynolds RJ (2008). Pollinator specialization and the evolution of pollination syndromes in the related Silene, S. caroliniana, S. virginica, and S. stellata. University of Maryland.

Reynolds RJ, Dudash MR, Fenster CB (2010). Multiyear study of multivariate linear and nonlinear phenotypic selection on floral traits of hummingbird-pollinated Silene virginica. Evolution 64: 358-369.

Reynolds RJ, Kula AAR, Fenster CB, Dudash MR (2012). Variable nursery pollinator importance and its effect on plant reproductive success. Oecologia 168: 439-448.

Reynolds RJ, Westbrook MJ, Rohde AS, Cridland JM, Fenster CB, Dudash MR (2009). Pollinator specialization and pollination syndromes of three related North American Silene. Ecology 90: 2077-2087.

Ritland K (1996). A Marker-Based Method for Inferences About Quantitative Inheritance in Natural Populations. Evolution (N Y) 50: 1062-1073.

Rodzen JA, Famula TR, May B (2004). Estimation of parentage and relatedness in the polyploid white sturgeon (Acipenser transmontanus) using a dominant marker approach for duplicated microsatellite loci. Aquaculture 232: 165182 .

Steven JC, Delph LF, Brodie ED (2007). Sexual dimorphism in the quantitative-genetic architecture of floral, leaf, and allocation traits in Silene latifolia. Evolution (N Y) 61: 42-57.

Strauss SY, Whittall JB (2006). Non-pollinator agents of selection on floral traits. In: Ecology and evolution of flowers, Oxford University Press: Oxford,UK Vol 1, pp 120-138.

Therneau TM, Sinnwell J (2015). kinship2: Pedigree Functions.

Wang J, Scribner KT (2014). Parentage and sibship inference from markers in polyploids. Mol Ecol Resour 14: 541553 .

Willis JH, Coyne JA, Kirkpatrick M (1991). Can One Predict the Evolution of Quantitative Characters Without Genetics? Source Evol Evol 45: 441-444.

Wilson AJ, Reale D, Clements MN, Morrissey MM, Postma E, Walling CA, et al. (2010). An ecologist's guide to the animal model. J Anim Ecol 79: 13-26.

Zhou J, Dudash MR, Fenster CB (2016). Cannibalism during early larval development of Hadena ectypa Morrison (Lepidoptera: Noctuidae). Proc Entomol Soc Washingt 118: 450-455.

Zhou J, Dudash MR, Fenster CB, Zimmer EA (2016). Development of highly variable microsatellite markers for the tetraploid Silene stellata (Caryophyllaceae). Appl Plant Sci 4: 1600117-1600117.

Zhou J, Dudash MR, Zimmer EA, Fenster CB (2018). Comparison of population genetic structures of the plant Silene stellata and its obligate pollinating seed predator moth Hadena ectypa. Ann Bot 122: 593-603.

Zhou J, Reynolds RJ, Zimmer EA, Dudash MR, Fenster CB (2020). Variable and sexually conflicting selection on Silene stellata floral traits by a putative moth pollinator selective agent. Evolution (N Y) 74: 1321-1334.

Zhou J, Zimmer EA, Fenster CB, Dudash MR (2018). Characterization of the mating system of a native perennial tetraploid herb, Silene stellata. Am J Bot 105: 1643-1652. 